\title{
Centralized Modeling of the Communication Space for Spectral Awareness in Cognitive Radio Networks
}

\author{
Jens P. Elsner, Christian Koerner and Friedrich K. Jondral \\ Universität Karlsruhe (TH), Germany, \{elsner, koerner, fj\}@int.uni-karlsruhe.de
}

The communication space is five dimensional: its degrees of freedom are frequency, time and space. The use of the electromagnetic spectrum depends on these parameters. With future applications such as opportunistic overlay access or distributed spectrum monitoring in mind, it is important to estimate the state of the communication space on the basis of incomplete or imprecise information. A promising approach are technology centric Cognitive Radio networks. In these networks, nodes cooperate to infer information on spectral occupancy. This conceptual paper proposes a novel approach for centralized modeling of the communication space with emphasis on spatial dependencies through the use of a regression model. The modeling approach is verified with practical measurements.

\section{Introduction}

The five dimensional communication space is spanned by the dimensions frequency, time and space ${ }^{1}$. The usage of the natural resource electromagnetic spectrum varies with these parameters. Access to spectrum is heavily regulated in all parts of the world. Traditionally, the access rules governing spectrum usage are formulated in terms of frequency ranges, largely ignoring the two remaining degrees of freedom: time and space. This historical approach to licensing has lead to inefficient use of spectrum. E.g., [2] reports an average load of 5.2 percent in the $3-3000 \mathrm{MHz}$ range, leaving a lot of room for improvement.

To increase the efficiency in spectrum usage, transmissions of an overlay system can take place in transmission opportunities or spectrum holes [3, 4]. Transmission opportunities are unused regions of the communication space spanned by time, frequency and location. The detection of spectrum holes has to be accurate to decrease the probability of interference with other systems. Due to the nature of measurements, identification of transmission opportunities is always based on incomplete and imprecise information. Conceptual challenges in radio networks such as the hidden node and exposed node problem suggest the use of cooperative identification of transmission opportunies, i.e., information sharing between wireless terminals.

A centralized modeling approach is considered in this paper: wireless terminals gather local information

\footnotetext{
${ }^{1}$ This work has been presented in part at the IEEE Workshop on Cognitive Radios, Las Vegas, USA, January 2008 [1]. It has been expanded to include practical measurement and regulatory considerations, a conceptual engine description and extended results.
}

on their environment (e.g. spectrum usage, location) and forward this information to a centralized instance, termed network. The network uses this information from distributed nodes to build an online model of the communication space state. This spectral awareness can then be used to optimize resource allocation.

The remainder of this paper is structured as follows: after a brief conceptual introduction to spectral awareness and its applications in this Section, Section II describes capabilities and requirements for spectral estimation in a Cognitive Radio (CR) terminal. Section III covers centralized modeling of the communications space. Concluding remarks and a summary of important research problems form Section IV.

\section{I.A. Scenario}

We consider a distribution of wireless nodes connected to a central instance. This central instance is referred to as network. The scenario is depicted in Figure 1. All CR terminals are assumed to have an out-of-band connection to the network ${ }^{2}$. Furthermore, the network is aware of the position of each CR terminal. This location awareness is a precondition for modeling of spatial dependencies and currently under investigation by numerous researchers $[4,5]$.

During operation, the $\mathrm{CR}$ terminals report information relevant to the state of the communication space to the network. This relevant information is an estimate of the spectral environment and an estimate of the current CR terminal position, which the network tracks. Based on the reported information, the network builds a model of the communication

\footnotetext{
${ }^{2}$ The nature of this feedback channel is not considered here and is in fact a fundamental conceptual issue of CR networks [4] 


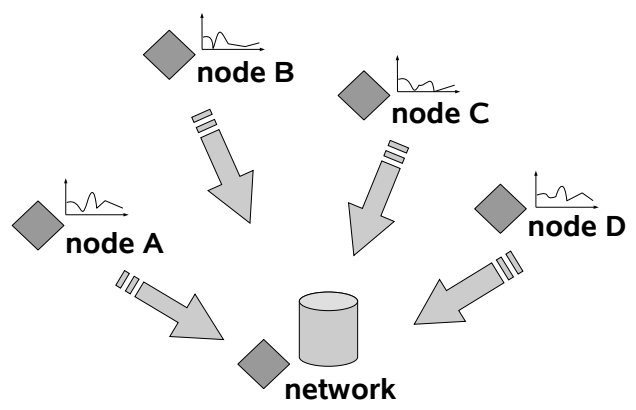

Figure 1: Principle of operation. Each node reports an estimate of its spectral environment to a centralized instance. The network is aware of the location of each of the nodes.

space. The resulting model is a physical representation of spectrum usage ("At a given position, what is the signal level in a given bandwidth now?"), which can be transformed into a more abstract representation ("There is a GSM downlink operating in this frequency range.") with a-priori information. Modeling of the communication space leads to spectral awareness of the network, i.e., the the ability to determine spectral occupancy for a given geographic location, frequency, and time.

\section{I.B. Related Work}

Collaborative spectrum sensing has been proposed before as a method to optimize the free/in-use decision of a spatial distribution of nodes monitoring the same channel in fading conditions $[6,7,8,9]$. Location awareness and modeling of the actual physical signal levels play only a minor role in these publications; the overall decision is based on a combination of local hard free/in-use decisions.

Haykin also tackles the problem of radio-scene analysis by adapting a method from geostatistics [4]. He proposes multivariate spectral analysis of the interference temperature time series estimate of each terminal to detect spectrum holes in a centralized manner, however, measurements or simulations to back this approach are not supplied.

Ferris et al. suggest the simpler approach of using Gaussian process models to represent a given spatial signal strength distribution $[10,11]$. Extending on this approach, this paper proposes a regression model for radio-scene analysis in location-aware CR networks.

\section{I.C. Advantages of Centralized Model- ing}

Minimization of mobile unit intelligence decreases power consumption, required computational resources and cost. Implementing intelligent behaviour at the mobile terminal is prone to be more expensive than comparable methods in the network, where computational power is virtually unlimited.

To minimize the amount of data transfered over the feedback channel to the network a certain amount of signal processing intelligence is needed at the terminals. The data describing the local spectral environment need to be reduced to a small symbolic representation. Finding representations and models that offer a good compromise in the trade-off between reduction of power consumption and computational resources, and hence data reduction, is important. A simple representation based on a quantized PSD estimate is proposed in Section II.B.

\section{I.D. Regulatory Considerations and Added Value}

Access to spectrum is strictly regulated in most countries of the world and subject to international laws and agreements. A politically acceptable general overlay access system will most likely have to assure complete operator control $[12]^{3}$.

A possibility to realize such a system is a network of overlay spectrum access stations (shown in Figure 2 ), run by the respective regulatory agency or subcontracted to a cellular operator, which covers the area of interest. These stations act as sensors for spectral awareness. The network operator or regulatory agency can then monitor the whole network and assign or revoke access rights dynamically to assure optimal spectrum utilization and minimize unsolicited interference. Primary users can create a secondary spectrum market by charging a fee for granting overlay access. In view of the potential for improvement [2], significant added value can be generated.

\section{Estimation of Spectral Occu- pancy at the CR Terminal}

Before turning to centralized spatiotemporal modeling, the measurement capabilities of the wireless terminal have to be defined. Modeling of the communication space is modeling of physical quantities, hence

\footnotetext{
${ }^{3}$ In [12], Mangold et al. evaluate a simple overlay concept based on a grant and denial beacon. They thoroughly motivate operator assisted dynamic spectrum assignment.
} 


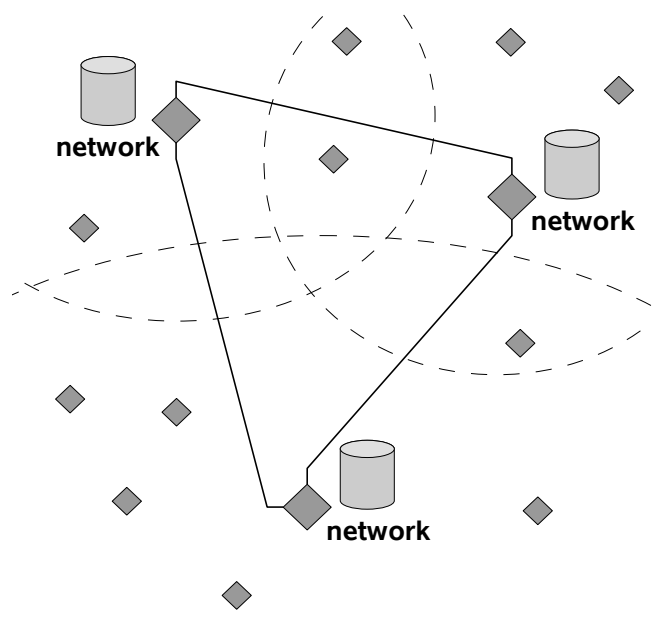

(a) Distributed centralized modeling in a overlay access network. A model is run for each area of interest. The spectrum access stations act as sensors for the spectral environment and collect information from the CR terminals.

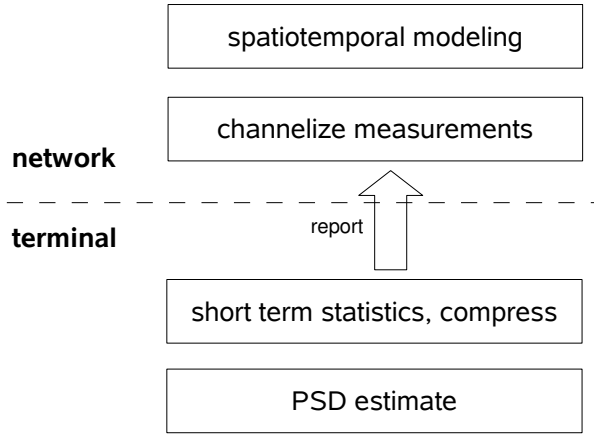

(b) Principle steps from spectral estimation to centralized model building. At the terminal, the PSD estimate is compressed (e.g. by channel segmentation, quantizing) and then reported to the network. The network collects the measurements and aggregates them in discrete channels. Each channel is then modeled separately.

Figure 2: Principle of operation

measurements of the CR terminal have to be made independent of a specific wireless standard. This implies a three step process at the CR terminal shown in Figure 2.

In a first step, the CR terminal node measures a signal level, possibly making use of a-priori information (matched filtering, feature detection). In a second step, this information is aggregated, compressed and then, in a third step, forwarded to the network. The network then uses this data to model the communication space occupancy.

The most general measurement step is spectral estimation, an energy based detection strategy.

\section{II.A. Spectral Estimation}

Spectral estimation, also known as spectral analysis or spectrum sensing in a CR context, is the simplest standard independent detection strategy. The measurement capabilities of a CR terminal with spectrum estimation comprise three basic steps:

1. Appropriate spectral estimation with respect to time and frequency resolution,

2. (optional) channel segmentation,

3. spectrum estimate compression (e.g. through quantization) and reporting.

\section{Maximum Hold}

For inherently instationary signals such as communication signals, spectral estimation algorithms can underestimate the maximum signal level in a given frequency range. E.g., if we choose to average periodograms over the duration of a whole GSM time division multiple access (TDMA) frame with only one active burst (cf. Figure 3 and 5), the resulting PSD estimate significantly underestimates the maximum received power. A comparison with a peak-hold estimate serves as an indicator that the averaging duration is too long: the estimated variance is higher than the expected variance for a given algorithm. This fact can be exploited to adapt spectral resolution, temporal resolution and window length to the data to get a more accurate maximum power estimate.

\section{Time - Frequency Resolution}

Spectral estimation methods have an inherent tradeoff between time resolution and frequency resolution. Choosing a long averaging duration results in good frequency resolution but poor localization of frequencies in time. A short duration on the other hand gives poor frequency resolution but good localization of frequencies. This is illustrated in Figure 3, a snapshot of a GSM1800 downlink band. With high temporal resolution the burst structure and power control is clearly visible but the channel bandwidth is overes- 


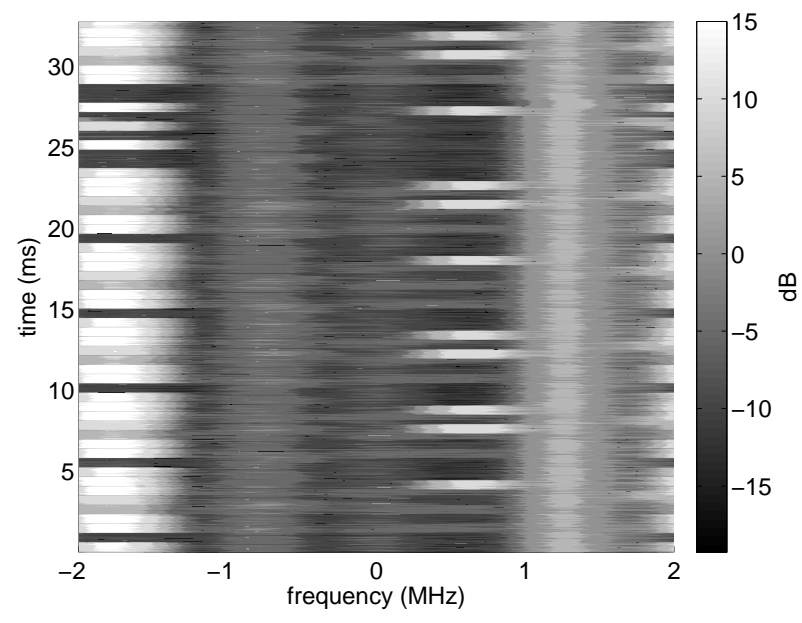

(a) High temporal resolution

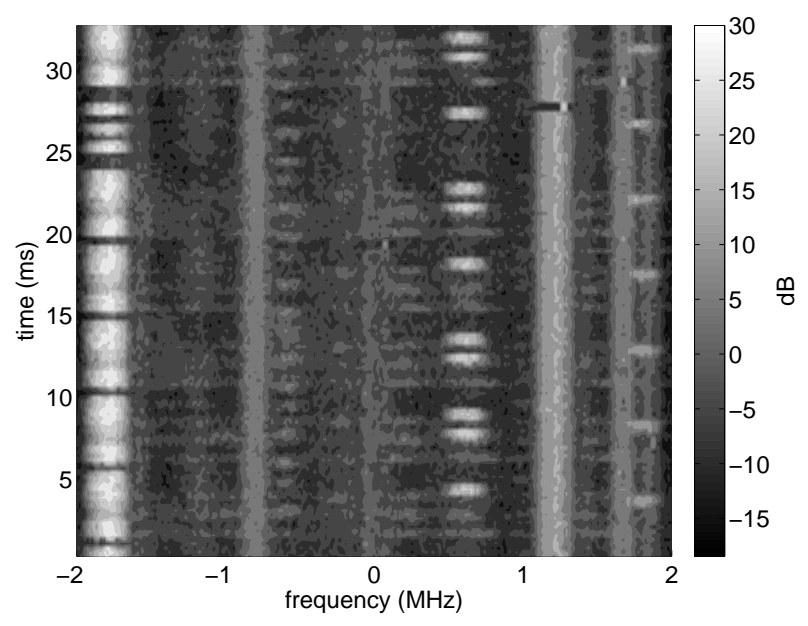

(b) High frequency resolution

Figure 3: Illustration of the influence of the time and frequency resolution choice. The time-frequency plane shown has a center frequency within the GSM 1800 band at $4 \mathrm{MHz}$ bandwidth. Both planes were computed from 131072 samples based on the Welch method with a Hamming window, yielding a frequency-time resolution of $64 \times 2048$ bins in Figure 3(a) and $2048 \times 64$ bins in Figure 3(b). Note the frequency leakage in Figure 3(a).

timated due to leakage. Choosing a long averaging duration and hence a high frequency resolution leads to a good estimate of the channel bandwidth while the burst structure is blurred. Furthermore, both time and frequency resolution can be traded against accuracy of the estimate.

\section{II.B. Data Representation and Report- ing}

The output of the spectral estimation stage is a quantized PSD, essentially a description of channels and signal levels. Figure 4(b) shows such an estimate. Optional channel segmentation can increase the accuracy of the PSD estimate, as quantization for data compression prior to reporting to the network can then be carried out more exactly. With the prior knowledge of the matched filters, the node can precisely report a single power estimate per channel and time slot.

\section{Centralized Modeling of the Communication Space}

The state of the communication space is the spatiotemporal distribution of signal levels in units of Watt per Hertz: loosely speaking, a spatial generalization of the power spectral density. An equivalent metric, interference temperature, has been proposed by the FCC Spectrum Policy Task Force [13]. It is the choice of the designer of the CR terminal whether to use the spectrum estimation/energy detection, equivalent to the interference temperature metric, or methods with increased sensitivity incorporating prior knowledge such as matched filtering. The following spatiotemporal model has to be independent of the power measurement method chosen.

\section{III.A. Physical Properties of the Wire- less Communication Channel}

The physics of wave propagation, i.e., phenomena such as attenuation, refraction and reflection, lead to spatial and temporal dependencies of the electromagnetic field strength.

For any given center frequency and receiver location, two stochastic phenomena dominate the spatiotemporal characteristics of the receiver signal level, i.e., the absolute values of the complex voltages at the receiver: fast fading and slow fading, also known as shadow fading. A third factor, long-distance variation of the signal level, can be regarded as deterministic and is described by the path loss between transmitter and receiver.

Fast fading is the location-independent fast variation of the signal amplitude and phase, attributed to varying multi-path propagation due to moving scatteres and receiver movements in the order of a wavelength [14]. The influence of fast fading on the signal is usually modeled by a Rayleigh or Rice amplitude distribution [15].

Slow fading is the variation of the local mean power over distances in the order of tens of wavelengths ${ }^{4}$.

\footnotetext{
${ }^{4}$ Salo et al. [16] distinguish between local shadow fading and global shadow fading. According to Salo, local shadow fading
} 


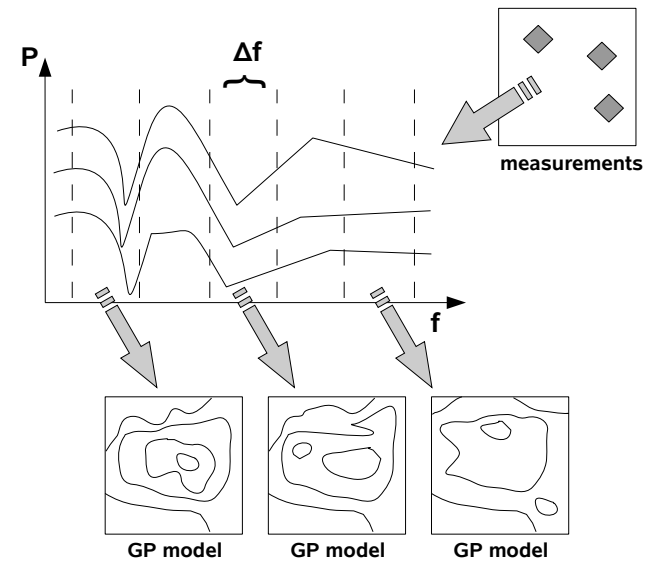

(a) Engine principle. A GP model is run for every frequency bin to build a complete spatiotemporal model of all frequencies in question.

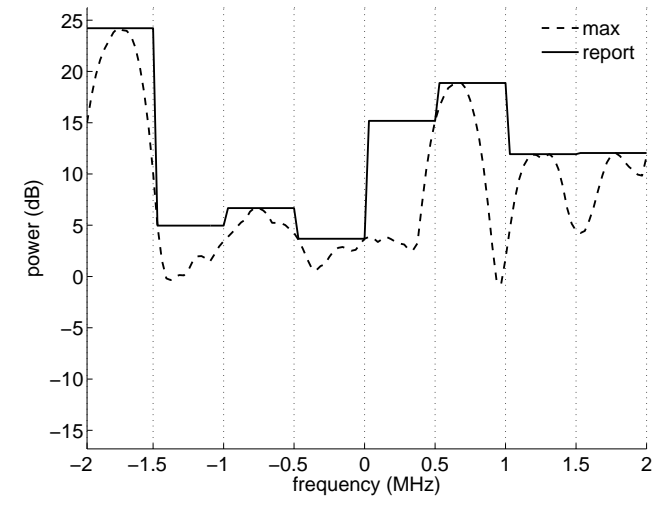

(b) Report for one measurement cycle: a discretized maximum PSD estimate with a frequency resolution of $500 \mathrm{kHz}$.

Figure 4: A model is run for every frequency bin

Slow fading can be modeled as a lognormal distributed amplitude [14].

Provided that the transmitter and receiver specific transmission characteristics (transmitter and receiver position, power etc.) remain unchanged, the local long-term average signal level at a given position can be regarded as constant as it changes slowly over long periods of time. In wave propagation modeling this local long-term average is calculated from the path loss and the antenna characteristics.

The path loss between two locations depends on distance and attenuation of the transmission environment and varies only with large-scale environmental changes.

To build an accurate generalizable model based on distributed measurements, these measurements have to be made independent of the actual receiver structure. Two significant factors limiting sensitivity are the individual antenna characteristics and noise figures. For a mobile receiver antenna orientation is usually random. Considering only the maximum gain of an antenna is not enough to characterise its performance in practical operating conditions [17]. Measurements are needed to determine the mean effective gain of a test antenna. The mean effective gain is defined as the ratio of the mean signal levels at the test antenna and a reference antenna $[17,18]$. In [17]

is the lognormal variation of the received signal amplitude in a area where path loss is constant. Over distances where path loss is not constant, the characteristics of shadow fading (mean and variance) change as well: this is termed global shadow fading. Marsan et al. [14] use the terms large area lognormal shadowing and small area lognormal shadowing.
Table 1: Wireless channel phenomena

\begin{tabular}{|c|c|}
\hline Type & Effects on signal level \\
\hline fast fading & $\begin{array}{c}\text { fast temporal variations, } \\
\text { statistics change with } \\
\text { velocity and location }\end{array}$ \\
\hline slow fading & $\begin{array}{c}\text { slow temporal variations, } \\
\text { statistics change spatially }\end{array}$ \\
\hline path loss & $\begin{array}{c}\text { deterministic spatial } \\
\text { variations }\end{array}$ \\
\hline $\begin{array}{c}\text { antenna characteristics, } \\
\text { orientation }\end{array}$ & fariation \\
\hline
\end{tabular}

Kalliola et al. analyse the effective gain in various indoor and outdoor scenarios. Mean effective gain values between $-5 \mathrm{dBi}$ for free space and $-11 \mathrm{dBi}$ for operation beside a head model are reported. This effective loss has to be accounted for in distributed measurements so that spectrum occupancy is not underestimated. The individual noise figure of a receiver limits sensitivity, but does not affect the validity of the model as long as physical quantities are reported.

\section{III.B. Parametric versus non- parametric modeling}

Traditional wave propagation modeling uses a parametric model of the communication space. A parametric communication space model has the advantage of representing the physical reality closely, its param- 
eters are number, position and power of transmitters, antenna characteristics and wave propagation environment. If these parameters are known, techniques from wave propagation modeling can be applied to make coverage predictions. Standard approaches include simple path loss calculations, simplified diffractionbased methods, ray tracing and field theoretic calculations [15]. The type of model used in a given application depends heavily on frequency, desired accuracy and terrain at hand. Sophisticated models use terrain information for relatively precise predictions. Unfortunately, even with these models the standard deviation of the prediction error is in the order of $10 \mathrm{~dB}$ [19] as the physical reality contains too many unknown factors (e.g. metal contents of buildings, non-stationary environmental influences such as traffic and weather conditions) to be precise. In practice, predictions are verified and fine-tuned with measurements [19]. This shows that even very complex models represent the physical environment inadequately. A parametric communication space model would have to estimate the right combination of number of transmitters, antennae characteristics etc. from an infinite number of possibilities. Without a-priori information this is infeasible - a communication space model relying on measurements only is bound to be nonparametric ${ }^{5}$.

\section{III.C. Choosing effects to model}

The spectral estimation step proposed in Section II results in a PSD estimate. The PSD does not include phase information. Accordingly, we are only interested in modeling receiver signal levels, i.e., the absolute values of the complex voltages at the receiver antenna, and not the complete channel impulse response.

Table 1 shows the different wireless channel phenomena. The communication space model has to provide means to capture the most significant effects. To summarize: for a fixed location, there are variations in the signal level over time due to fading and transmitter activity. For a fixed moment in time, there are spatial variations in the signal level due to path loss.

The model should capture only spatiotemporal dependencies directly related to spectrum occupancy. Hence, we are interested in modeling deterministic spatial variations due to path loss, spatial statistical

\footnotetext{
${ }^{5}$ Even if a direct parametric modeling approach seems infeasible, it is possible to infer information about locations and number of transmitters and transmission characteristics in a second step. A parametric representation of the cause of the communication space state can be built on top of the non-parametric effect model: this of interest when monitoring unsolicited interference.
}

variations due to slow fading on top of path loss, and transmitter activity. Fast fading is eliminated at the $\mathrm{CR}$ terminal during measurement by averaging.

The concrete local long-term average of the receive power $P_{\mathrm{R}}\left(\mathrm{x}_{\mathrm{R}}\right)^{6}$ at the receiver location is calculated with the radiated equivalent isotropic radiated power $P_{\mathrm{T}}\left(\mathrm{x}_{\mathrm{T}}\right)$, transmitter antenna gain $G_{\mathrm{T}}$, receiver antenna gain $G_{\mathrm{R}}$ and path loss $D\left(\mathbf{x}_{\mathrm{T}}, \mathbf{x}_{\mathrm{R}}\right)$, depending on both receiver and transmitter location:

$$
\begin{aligned}
P_{\mathrm{R}}\left(\mathbf{x}_{\mathrm{R}}\right)= & P_{\mathrm{T}}\left(\mathbf{x}_{\mathrm{T}}\right)+G_{\mathrm{T}}+G_{\mathrm{R}} \\
& -D\left(\mathbf{x}_{\mathrm{T}}, \mathbf{x}_{\mathrm{R}}\right)[\mathrm{dB}]
\end{aligned}
$$

The measured power at the receivers is equivalent to

$$
P_{\mathrm{M}}\left(\mathrm{x}_{\mathrm{R}}\right)=P_{\mathrm{R}}\left(\mathrm{x}_{\mathrm{R}}\right)-G_{\mathrm{R}}+D_{\mathrm{SF}}[\mathrm{dB}],
$$

where $P_{\mathrm{R}}\left(\mathrm{x}_{\mathrm{R}}\right)-G_{\mathrm{R}}$ is the variable to be modeled in this work and $D_{\mathrm{SF}}$ denotes slow fading and is equivalent to the measurement noise.

To account for different antenna characteristics, it is assumed that all measurements are normalized according to the mean effective gain of the receiver antenna.

The goal of the model is to capture the effects, not the cause of spatial spectrum occupation. Separating the effects from the cause allows for non-parametric modeling.

\section{Spatial Dependencies}

Measurements are only available for distinct locations. The model should make resonable predictions for locations without measurements, interpolating from available data. A good interpolation method should also provide a measure of reliability. The model should capture deterministic spatial variations in a path loss component and spatial statistical variations caused by slow fading.

Gaussian process regression, also known as kriging $^{7}$, is a modeling method from geostatistics. Using Gaussian processes (GPs) as a statistical nonparametric model offers an elegant way to represent location-dependent spectrum usage.

A key question to be answered is that of spatial correlation between measurements, i.e., the question of relevancy of observations in one location to neighboring locations. Spatial correlation of signal strength measurements has been studied extensively in literature, c.f. $[21,22]$ and references therein. These studies aim at describing the stochastic properties of signal strength distribution to develop suitable wireless

\footnotetext{
${ }^{6}$ Bold script denotes a collection of variables or a vector.

${ }^{7}$ Named after South African mining engineer D. G. Krige [20].
} 
channel simulations for different propagation environments. Extending this idea, it is possible with georeferenced statistics, i.e., location aware $\mathrm{CR}$ terminals, to embed measurements into a stochastic framework.

\section{III.D. Regression with Gaussian Pro- cesses}

A Gaussian process (GP) is a set of random variables denoted by $f\left(\mathbf{x}_{i}\right)$, any finite number of which have a jointly Gaussian distribution ${ }^{8}$ [20].

The value of the underlying latent function at a location $\mathbf{x}_{i}$ is represented by the GP $f\left(\mathbf{x}_{i}\right)$. A GP is defined by its mean

$$
m(\mathbf{x})=\mathrm{E}\{f(\mathbf{x})\}
$$

and covariance function $\operatorname{cov}\left(f\left(\mathbf{x}_{p}\right), f\left(\mathbf{x}_{q}\right)\right)=$ $k\left(\mathbf{x}_{p}, \mathbf{x}_{q}\right)$,

$k\left(\mathbf{x}_{p}, \mathbf{x}_{q}\right)=\mathrm{E}\left\{\left(f\left(\mathbf{x}_{p}\right)-m\left(\mathbf{x}_{p}\right)\right)\left(f\left(\mathbf{x}_{q}\right)-m\left(\mathbf{x}_{q}\right)\right)\right\}$.

Without loss of generality, the mean function can be assumed to be zero. If the covariance function is properly defined ${ }^{9}$, a GP can be used as a non-parametric model for machine learning: an observation $\left(y_{i}, \mathbf{x}_{i}\right)$ is thought to be related to the random variable $f\left(\mathbf{x}_{i}\right)$ modeling a latent function. With a signal model and a given set of $n$ observations $D=\left\{\left(y_{i}, \mathbf{x}_{i}\right) \mid i=\right.$ $1, \ldots, n\}$, the model calculates the posterior distribution of the GP at a test point.

\section{Predictions with Noisy Observations}

The signal model is defined as

$$
y=f(\mathbf{x})+\varepsilon,
$$

where $\varepsilon$ is additive independent Gaussian noise with variance $\sigma_{n}^{2}$. Taking into account the additive Gaussian noise, the covariance between the observations can be written as

$$
\operatorname{cov}\left(y_{p}, y_{q}\right)=k\left(\mathbf{x}_{p}, \mathbf{x}_{q}\right)+\sigma_{n}^{2} \delta_{p q}
$$

where $\delta_{p q}$ denotes the Kronecker delta which is one iff $p=q$ and zero otherwise.

The $n \times n$ covariance matrix $K_{y}$ of a set of noisy observations is then

$$
K_{y_{p, q}}=\operatorname{cov}\left(y_{p}, y_{q}\right) .
$$

\footnotetext{
${ }^{8} \mathrm{We}$ follow the function-space view of Rasmussen and Williams [20].

${ }^{9}$ The resulting covariance matrix needs to be invertible [20].
}

Let $\mathbf{k}\left(\mathbf{x}_{*}\right)$ be the $n \times 1$ vector of covariances between the training points, i.e., locations at which observations were obtained, and an arbitrary test point $\mathbf{x}_{*}$ :

$$
\mathbf{k}\left(\mathbf{x}_{*}\right)=\left[k\left(\mathbf{x}_{\mathbf{1}}, \mathbf{x}_{*}\right), \ldots, k\left(\mathbf{x}_{\mathbf{m}}, \mathbf{x}_{*}\right)\right]^{T} .
$$

The conditional mean with zero prior mean $m(\mathbf{x})$ and variance at the test point are then calculated as [20]

$$
\mu_{\mathbf{x}_{*}}=m_{*}\left(\mathbf{x}_{*}\right)=\mathbf{k}\left(\mathbf{x}_{*}\right)^{T} K_{y}^{-1} \mathbf{y}
$$

and

$$
\sigma_{\mathbf{x}_{*}}^{2}=k_{*}\left(\mathbf{x}_{*}, \mathbf{x}_{*}\right)=k\left(\mathbf{x}_{*}, \mathbf{x}_{*}\right)-\mathbf{k}_{*}^{T} K_{y}^{-1} \mathbf{k}_{*} .
$$

The regression model is given by (9) and (10). To make predictions using the model an appropriate covariance function has to be specified. This covariance function is application specific. The principal shape of the covariance function is chosen based on a-priori knowledge. The shape still has remaining degrees of freedom, the so-called hyperparameters. These hyperparameters are estimated from the measurement data as a whole. For a spatial signal strength distribution model, $y_{i}$ is a measurement at a location $\mathbf{x}_{\mathbf{i}}$. Taking the stochastic properties of shadow fading into account, an exponential covariance function is an appropriate choice for modeling two dimensional spatial data as shown by Giancristofaro and Gudmundson $[22,23]$ :

$$
\begin{gathered}
k\left(\mathbf{x}_{i}, \mathbf{x}_{j}\right)=\sigma_{f}^{2} \exp \left(\left(\mathbf{x}_{j}-\mathbf{x}_{i}\right) \Sigma\left(\mathbf{x}_{j}-\mathbf{x}_{i}\right)^{T}\right)+\sigma_{n}^{2} \delta_{p q} \\
\text { with } \quad \Sigma=\operatorname{diag}\left(1 / l_{x}^{2}, 1 / l_{y}^{2}\right) .
\end{gathered}
$$

A stochastic process with this covariance function is stationary in space, as it is only a function of the distance of two locations $\left|\mathbf{x}_{\mathbf{j}}-\mathbf{x}_{\mathbf{i}}\right|$. The squared exponential covariance has different characteristic lengths $l_{x}$ and $l_{y}$, allowing different correlation properties on the $x$ and $y$ scale. $\sigma_{f}$ is the GP model standard deviation, $\sigma_{n}$ is the standard deviation of the measurement noise. All hyperparameters $\Theta=\left[l_{x}, l_{y}, \sigma_{f}^{2}, \sigma_{n}^{2}\right]^{T}$ need to be estimated from the data. The maximum likelihood estimate is given by maximizing the likelihood $\log p(\mathbf{y} \mid D, \boldsymbol{\Theta})$ or equivalently by minimizing the objective function

$$
f=\log \left|K_{y}\right|+\mathbf{y}^{T} K_{y}^{-1} \mathbf{y}
$$

where $\mathbf{y}$ is the vector of all observations. This optimization problem is efficiently solvable by conjugate gradient descent [20]. 


\section{Interpretation of Model Noise and Exem- plary Measurements}

Figure 6 shows the result of GP regression for a channel of the GSM900 band in contour plots ${ }^{10}$. The data is displayed for a varying number of measurements, made in the GSM900 bands and georeferenced with GPS accuracy. Areas in which the estimated cumulative model and noise standard deviation uncertainty exceeds $6 \mathrm{~dB}$ are not plotted. In these areas, only few measurements were made and hence the prediction is uncertain. As slow fading is lognormal distributed, the GP model works on logarithmic measurement data. The prior mean function is assumed to be zero, $m(\mathbf{x})=0$; the measurement data are centered to account for this assumption. $\sigma_{n}^{2}$ describes the model noise and the variance of lognormal fading. The location-independent fast fades are averaged out during the spectrum estimation and reporting step.

\section{III.E. Extension to Temporal Dependen- cies}

The GP model for spatial dependencies can easily be generalized to include temporal dependencies. One has to differentiate between long term dependencies, which are covered by the model, and short term dependencies of the communication space which are not included. This has a very practical reason: short term dependencies cannot be modeled in a centralized manner as this information is lost during the sporadic reporting stage. Furthermore, the model describes only the state of the communication space over, e.g., the last few minutes. This state can be regarded as current, but it does provide information about future spectrum usage. Obviously then, short term communication space changes have to be dealt with at the terminal.

The centralized model has a temporal resolution appropriate to model longer term dependencies. With respect to overlay system use, this approach can be used to opportunistically reuse completely unused frequency ranges, which tend to stay free in certain areas for longer periods of time.

\section{Long term dependencies}

The GP model can be generalized to include long term dependencies by temporal windowing of measurement data. The underlying assumption, in analogy to spectral estimation, is that the latent function modeled by GP regression can be treated as stationary in the window duration.

\footnotetext{
${ }^{10} \mathrm{~A}$ verification of the model and further details can be found in [1]
}

If only limited measurements are available, the stationarity requirements can be relaxed if the wireless terminals report only the maximum of all PSD estimates to the network. Then, only the maximum signal level for each channel has to be stationarity. This eliminates the influence of sporadic waveforms and separates long-term from short-term dependencies. An example is shown in Figure 5(b).

\section{Short term dependencies}

Short term dependencies are communication space changes not covered by the proposed GP model. Short term dependencies are, for example, the TDMA burst structure in a GSM system as shown in Figure 5(a) or the periodic bursty nature of a RADAR sweep.

In an overlay context, modeling to exploit these small scale transmission opportunities has to be done directly at the terminal. Several approaches have been proposed in literature, e.g., Mangold et al. [24] suggest a histogram based approach, now part of the proposed IEEE $802.11 \mathrm{k}$ amendment [25].

If only long-term dependencies are modeled, it is not guaranteed that, due to the processing delay of the network, a frequency resource assigned for transmission by the network, is indeed available. An overlay terminal has to perform channel sensing regularly to detect previously inactive primary system transmitters. If an active primary transmission is detected, it has to back-off and report to the network.

\section{III.F. Model Engine Concept}

The spatial spectrum map, if available for different frequency ranges, can be used to identify transmission opportunities in frequency and space, i.e, unused frequency ranges or areas in which the signal level is below a threshold.

A complete model of the communication space can be constructed by running several regression models for different frequency ranges. The spatial resolution is not discretized a-priori but results from the defined test point grid. The frequency resolution is chosen smaller than the smallest channel bandwidth of interest. The time resolution is a compromise between number of measurements available and stationarity requirements.

For each frequency bin, a GP model is run, yielding a complete representation of the communication space. Figure 2 and Figure 4 illustrate the proposed concept. The estimation of hyperparameters can be based on the fact that the large scale environment is stationary and averaged over a long period of time. 


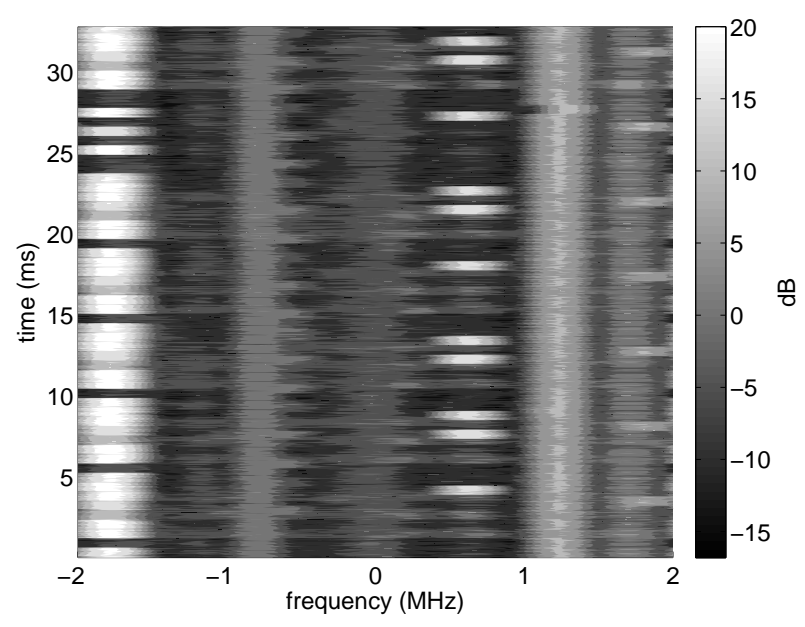

(a) Time-frequency plane

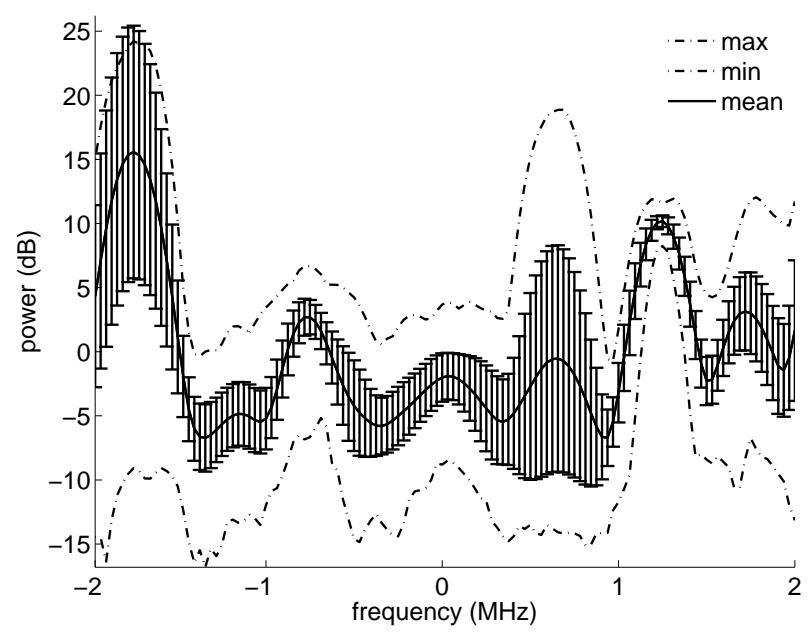

(b) statistics

Figure 5: Illustration of short term temporal dependencies in the GSM1800 band. The piece of spectrum analyzed is the same as in Figure 3, this time with a fitting time-frequency resolution (128 frequency bins). The burst characteristic is clearly visible. Figure $5(\mathrm{~b})$ shows the short term statistics (minimum, maximum and mean with estimated standard deviation) of the time-frequency plane.

GP regression requires the inversion of a covariance matrix. Matrix inversion is of complexity $O\left(n^{3}\right)$, which makes large models computationally prohibitive. However, GP regression with a stationary covariance functions can also be formulated as filtering with an equivalent kernel and is tantamount to Wiener filtering $[26,20]$. A practical implementation has to be based on this filtering approach due to performance reasons.

\section{Conclusion and Further Re- search}

Centralized modeling of the communication space is able to capture spatial and long term temporal variations of spectrum usage. Gaussian process based regression is an appropriate tool to capture these variations and provides a measure of confidence for the estimate. The regression is based on distributed cooperation of CR terminals, which use spectrum estimation to infer information about their spectral environment. The spectral estimation stage was described conceptually and the fundamental estimation problems stemming from applying stationary statistics to inherently unstationary temporal signals were highlighted.

Centralized modeling is a feasible solution to create spectral awareness in radio networks under the precondition of location awareness. The proposed model of the communication space can be used to improve spectral efficiency through overlay use of spectrum and demand assignment. At the same time it provides a means to monitor additional inferference.

\section{Research Issues}

The key preconditions upon which the proposed model is built are appropriate spectral estimation and location awareness ${ }^{11}$ at the terminal. Fitting algorithms for spectral estimation and channel segmentation in CR terminals, both in terms of complexity and accuracy, and radio-frontend design are still subject to research. Location estimation and tracking in CR terminals for indoor and outdoor localization without the use of an explicit positioning system is another research field where few concrete results are available. Promising is the adaptation from methods of autonomous robotics as proposed in [10] and [11].

The ad hoc concept of communication space introduced to motivate physical modeling should be put on sound mathematical footing. Generalizing the estimation theory by including a spatial dimension should yield more theoretical insight into the physics of communication and shed light on fundamental limitations $^{12}$.

\footnotetext{
${ }^{11}$ Location awareness does not only affect the physical layer. It has implications for privacy and security and offers new usercentric applications. An recent overview article about the potentials of location awareness is given by Celebi and Arslan [5].

${ }^{12}$ To the best of the author's knowledge, a comprehensive statistical description of the wireless channel, which includes temporal and spatial effects, is still missing. Such a model is necessary to justify all aspects of a non-parametric GP model theoretically. Georeferenced measurement campaigns could provide the necessary data to build such a model.
} 


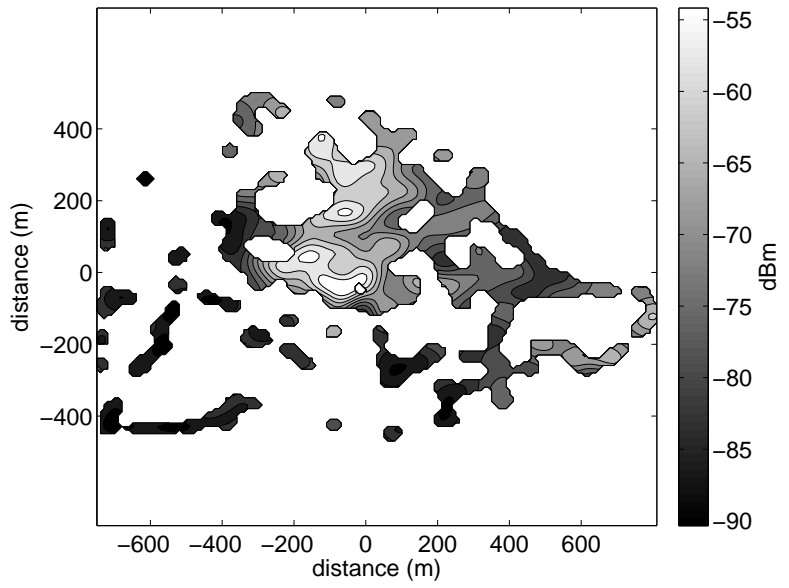

(a) 250 measurements

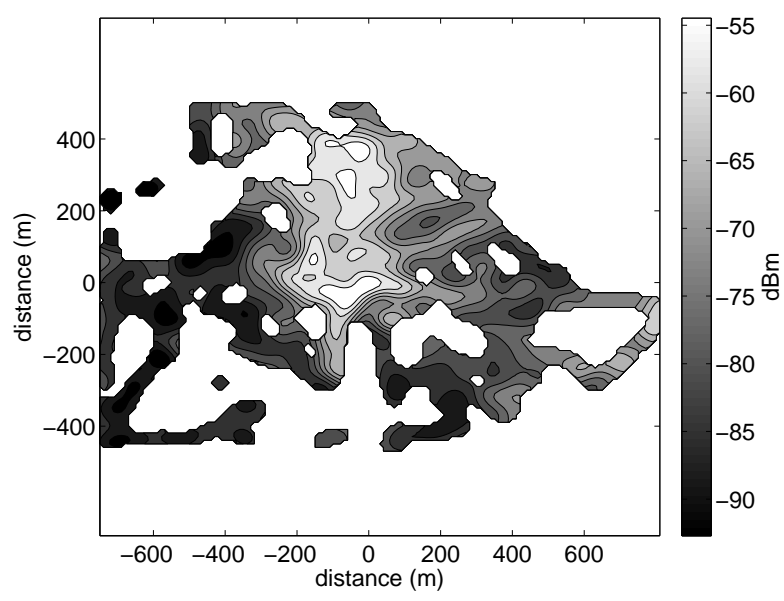

(c) 1000 measurements

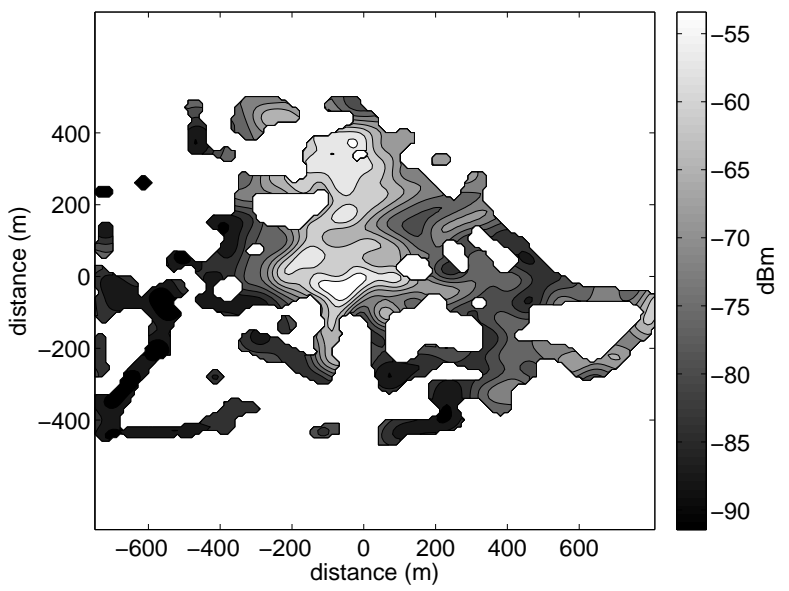

(b) 500 measurements

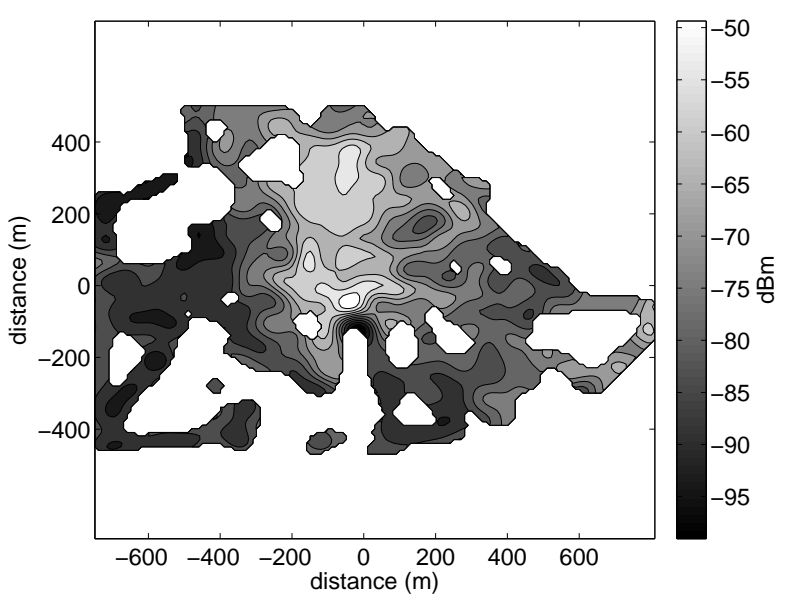

(d) 2500 measurements

Figure 6: These contour plots show GP regression for models of varying complexity and $2 \sigma<12 \mathrm{dBm}$, assuming $l_{x}=40 \mathrm{~m}, l_{y}=40 \mathrm{~m}$ with lognormal noise of $3 \mathrm{~dB}$ standard deviation. 
Last but not least the GP model itself has to be improved. Spatial non-stationary modeling is likely to be more accurate in scenarios with different types of environment [27]. Non-Gaussian noise models might help to increase the robustness of the model against outliers [28]. Another important open question is the robustness against positional noise, neglected in this study.

\section{References}

[1] J. P. Elsner, C. Körner, and F. K. Jondral, "Nonparameteric Modeling with Gaussian Processes for Spatial Radio-Scene Analysis," in Proceedings of the Consumer Communications and Networking Conference, Las Vegas, USA, January 2008.

[2] "Shared Spectrum Company," http://www.sharedspectrum.com.

[3] F. K. Jondral, "Software Defined Radio - Basics and Evolution to Cognitive Radio," EURASIP Journal on Wireless Communications and Networking, no. 3, pp. 275-283, 2005.

[4] S. Haykin, "Cognitive radio: brain-empowered wireless communications," IEEE Journal on Selected Areas in Communications, Feb. 2005.

[5] H. Celebi and H. Arslan, "Utilization of Location Information in Cognitive Wireless Networks," IEEE Wireless Communications, Aug. 2007.

[6] E. Visotsky, S. Kuffer, and R. Peterson, "On Collaborative Detection of TV Transmissions in Support of Dynamic Spectrum Sharing," Nov. 2005.

[7] A. Ghasemi and E. S. Sousa, "Collaborative Spectrum Sensing for Opportunistic Access in Fading Environments," Nov. 2005.

[8] S. M. Mishra, A. Sahai, and R. W. Brodersen, "Cooperative Sensing among Cognitive Radios," in IEEE International Conference on Communications, Jun. 2006.

[9] A. Ghasemi and E. S. Sousa, "Asymptotic Performance of Collaborative Spectrum Sensing under Correlated Log-Normal Shadowing," IEEE Communication Letters, Jan. 2007.
[10] B. Ferris, D. Hähnel, and D. Fox, “Gaussian Processes for Signal Strength-Based Location Estimation," in Proceedings of Robotics: Science and Systems, Philadelphia, USA, August 2006.

[11] B. Ferris, D. Fox, and N. Lawrence, "WiFiSLAM Using Gaussian Process Latent Variable Models," in Proceedings of the International Joint Conferences on Artificial Intelligence, Hyderabad, India, January 2007.

[12] S. Mangold, A. Jarosch, and C. Monney, “Operator Assisted Cognitive Radio and Dynamic Spectrum Assignment with Dual Beacons - Detailed Evaluation," in First International Conference on Communication System Software and Middleware, Jan. 2006.

[13] "Report of the Spectrum Efficiency Working Group," Federal Communications Commission - Spectrum Policy Task Force, Tech. Rep., Nov. 2002, eT Docket no. 02-135.

[14] M. J. Marsan, G. C. Hess, and S. S. Gilbert, "Shadowing Variability in an Urban Land Mobile Environment at $900 \mathrm{MHz}$," Electronics Letter, vol. 26, May 1990.

[15] N. Geng and W. Wiesbeck, Planungsmethoden für die Mobilkommunikation, Funknetzplanung unter realen physikalischen Ausbreitungsbedingungen. Springer, 1998, In German.

[16] J. Salo, L. Vuokko, H. M. El-Sallabi, and P. Vainikainen, "An Additive Model as a Physical Basis for Shadow Fading," IEEE Transactions on Vehicular Technology, Jan. 2007.

[17] K. Kalliola, K. Sulonen, H. Laitinen, O. Kivekäs, J. Krogerus, and P. Vainikainen, "Angular power distribution and mean effective gain of mobile antenna in different propagation environments," IEEE Transactions on Vehicular Technology, Sep. 2002.

[18] K. Ogawa, T. Matsuyoshi, and K. Monma, "An Analysis of the Performance of a Headset Diversity Antenna Influenced by Head, Hand, and Shoulder Effects at $900 \mathrm{MHz}$ : Part I - Effective Gain Characteristics," IEEE Transactions on Vehicular Technology, May 2001.

[19] B. Belloul and S. Saunders, "Improving predicted coverage accuracy in macrocells by use of measurement-based predictions," in Proceedings of the Twelfth International Conference on Antennas and Propagation, March 2003. 
[20] C. E. Rasmussen and C. K. I. Williams, Gaussian Processes for Machine Learning. The MIT press, 2006, available online: http://www.gaussianprocess.org/gpml/.

[21] A. Algans, K. I. Pedersen, and P. E. Mogensen, "Experimental Analysis of the Joint Statistical Properties of Azimuth Spread, Delay Spread, and Shadow Fading," IEEE Journal on Selected Areas in Communication, 2002.

[22] D. Giancristofaro, "Correlation model for shadow fading in mobile radio channels," Electronics Letter, May 1996.

[23] M. Gudmundson, "Correlation model for shadow fading in mobile radio channels," Electronics Letter, Sep. 1991.

[24] S. Mangold, Z. Zhong, K. Challapali, and C.-T. Chou, "Spectrum Agile Radio: Resource Measurements for Opportunistic Spectrum Usage," in Proceedings of the IEEE Global Telecommunications Conference (GLOBECOM), Dec 2004.

[25] "IEEE 802 LAN/MAN Standards Committee, 802.11 WG on WLANs," http://www.ieee802.org/11/.

[26] R. B. Gramacy, "Bayesian Treed Gaussian Process Models," Ph.D. dissertation, University of California Santa Cruz, 2006.

[27] R. B. Gramacy, H. K. H. Lee, and W. MacReady, "Parameter Space Exploration With Gaussian Process Trees," in Proceedings of the International Conference on Machine Learning. Omnipress and ACM Digital Library, 2004.

[28] M. Kuss, "Gaussian Process Models for Robust Regression, Classification, and Reinforcement Learning," Ph.D. dissertation, Technische Universität Darmstadt, 2006. 